\title{
WR-3 Band Waveguide Filter Tolerance Analysis and Surface Metallization Comparison
}

\section{Daniel Miek $^{1}$ (D) . Fynn Kamrath ${ }^{1} \cdot$ Patrick Boe $^{1} \cdot$ Michael Höft $^{1}$}

Received: 24 June 2020 / Accepted: 6 August 2020 / Published online: 22 August 2020

(C) The Author(s) 2020

\begin{abstract}
In this paper, a fifth-order WR-3 band waveguide filter with two transmission zeros (TZs) is presented. The design process is described and manufacturing issues are addressed. The filter is designed to fulfill a center frequency of $237.9 \mathrm{GHz}$ and a fractional bandwidth of $2.64 \%$. Three identical filters are manufactured with the classical computerized numerical control (CNC) milling technique in order to obtain information about the manufacturing repeatability for filter structures in the WR-3 band. The comparison shows that even in very high frequency regions a good repeatability can be achieved, if modern high-speed cutting (HSC) CNC milling machines are used. A comprehensive tolerance analysis is accomplished to classify the obtained results. The filters were manufactured from brass to ensure a meaningful comparability and due to the good machining qualities in CNC machines. In order to reduce the losses, the filters are afterwards sputtered with different materials (gold, silver, copper) to evaluate the effect on the insertion loss at very high frequencies. In comparison with the literature, it is shown that even narrow-band filters, which show a high sensitivity to machining tolerances, can be manufactured with high reliability and manufacturing repeatability in modern $\mathrm{CNC}$ milling machines. One of the measured filters shows a return loss higher than $20 \mathrm{~dB}$ over the whole passband.
\end{abstract}

Keywords Coupled triplet filter · J-band · H-plane split block · Waveguide filter · WR-3 band

Daniel Miek

dami@tf.uni-kiel.de

Fynn Kamrath

flk@tf.uni-kiel.de

Patrick Boe

pabo@tf.uni-kiel.de

Michael Höft

mh@tf.uni-kiel.de 


\section{Introduction}

Increasing data rates in modern communication systems lead to a steadily increase of the carrier frequency of such systems. While in the lower $\mathrm{GHz}$ range several techniques are available for the realization of bandpass filters, in sub-THz bands waveguide filters are the only meaningful solution due to size and loss issues.

The techniques for their realization are rather different as small sizes of the components lead to tight manufacturing constraints. Novel manufacturing techniques comprise the SU-8 photoresist technique $[3,16]$ as well as the deep reactive ion etching (DRIE) technique [8]. These techniques show a high potential, especially for smaller components in even higher frequency ranges. However, a common approach is to use classical computerized numerical control (CNC) milling machines as proposed by different groups in e.g. [5-7, 19]. The advantages of CNC milling techniques are the large flexibility of realizable geometries and the widespread use of this technology.

In this paper, three identical fifth-order WR-3 band filters comprised of two cascaded triplets are manufactured by a modern high-speed cutting (HSC) CNC milling machine. The measurement of the identically constructed filters provides insight about the expected manufacturing repeatability. A tolerance analysis is accomplished to empirically associate manufacturing inaccuracies (measured in root mean square (RMS) deviations) with an expected return loss. Finally, the effect of different surface materials on the filters insertion loss will be investigated.

This paper is organized as follows: The filter design and manufacturing issues are addressed in Section 2. To investigate the manufacturing repeatability of modern CNC milling machines, the filter was manufactured three times. The S-parameters are compared and examined in Section 3. Furthermore, a tolerance analysis is accomplished to link the manufacturing deviation to an expected return loss value in the passband. In Section 4, the filters were metallized with gold, silver, and copper. The effect on the insertion loss and the Q-factor is discussed. Section 5 compares the work proposed here with related works of other groups while Section 6 summarizes and concludes this paper.

\section{Filter Design and Manufacturing}

The aim of this paper is to investigate the manufacturing repeatability of modern HSC milling machines. Therefore, a filter design was chosen which can be realized in one component (i.e., alignment problems of different filter parts should be avoided as far as possible). As a further design constraint, the source and the load port should be separated from each other to avoid bending of the feed lines. The fractional bandwidth must not be chosen too high as manufacturing tolerances have a larger effect on narrow-band filters.

Therefore, the filter proposed here consists of five resonators and is designed as a cascaded triplet topology [2] in order to physically separate the source and load port. The filter specifications are set to a center frequency of $f_{0}=237.9 \mathrm{GHz}$ and a bandwidth of $B=6.27 \mathrm{GHz}(F B W=2.64 \%)$. Two TZs are foreseen below the 
passband at frequencies $f_{T z, 1}=229.5 \mathrm{GHz}$ and $f_{T z, 2}=232.8 \mathrm{GHz}$ (corresponding to normalized frequencies $f_{T z, L p, 1}=-2.73$ and $f_{T z, L p, 2}=-1.63$, respectively). The filter is matched to a return loss of at least $20 \mathrm{~dB}$ within the passband.

The filter topology was chosen as two cascaded triplets and is shown in Fig. 1 (c). The first triplet comprises resonators one to three while the second triplet consists of resonators three to five. Resonator three is therefore shared between both triplets. The filter design process in order to find the initial physical dimensions is accomplished conventionally: In the first step, the coupling matrix coefficients of the topology shown in Fig. 1 (c) are calculated based on the procedure described in [2]. The corresponding coupling coefficients to fulfill the specifications defined above are given in the caption of Fig. 1. The initial physical dimensions were subsequently found by calculating the coupling factor of sub-structures from the even and odd eigenmodes

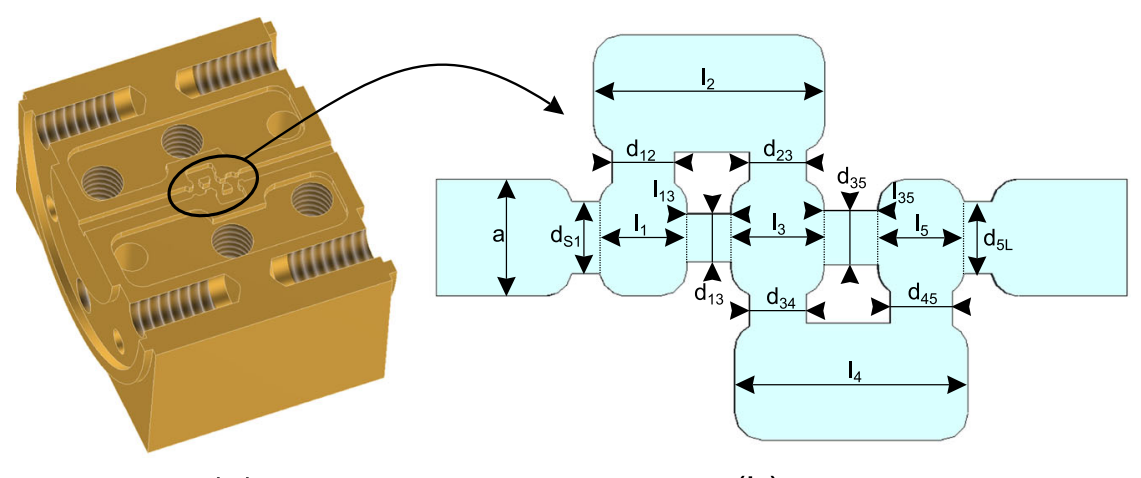

(a)

(b)

(S) Source port

i-th $\mathrm{TE}_{101}$ Resonator

i-th $\mathrm{TE}_{102}$ Resonator

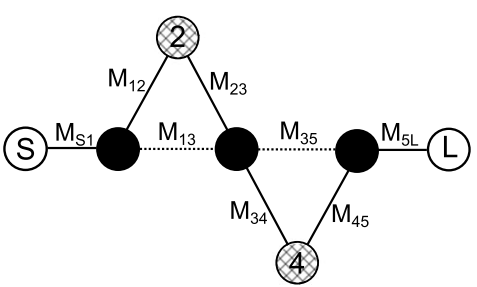

(c)

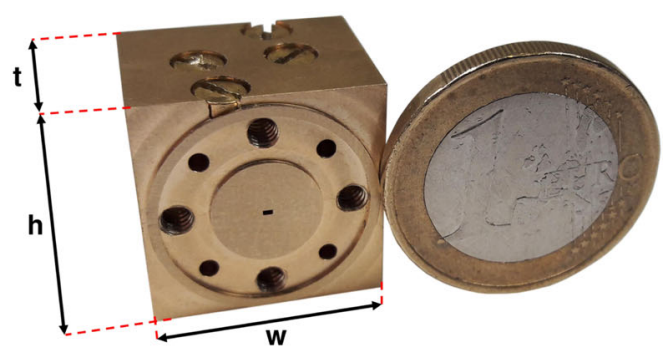

(d)

Fig. 1 (a) CAD model of the fifth-order filter, (b) principle drawing of the filter structure. The dimensions are $($ all in $\mu \mathrm{m}) d_{S 1}=537, d_{12}=457, d_{23}=419, d_{34}=421, d_{45}=455, d_{5 L}=537, d_{13}=361$, $d_{35}=402, l_{1}=649, l_{2}=1706, l_{3}=687, l_{4}=1721, l_{5}=635$. The waveguide channel has a width of $a=864 \mu \mathrm{m}$ and a unique depth of $432 \mu \mathrm{m}$. (c) Corresponding coupling topology with coefficients as follows: $M_{S 1}=1.014, M_{12}=0.843, M_{23}=0.612, M_{34}=0.552, M_{45}=0.781, M_{5 L}=1.014, M_{13}=$ $-0.207, M_{35}=-0.377, M_{11}=-0.04, M_{22}=0.246, M_{33}=-0.125, M_{44}=0.489, M_{55}=-0.04$. (d) Size comparison of a manufactured filter with a 1 Euro coin. The 1 Euro coin has a diameter of $23.25 \mathrm{~mm}$ while the dimensions of the split block are $w=h=20 \mathrm{~mm}, t=15 \mathrm{~mm}$ 
as comprehensively described in [9]. To ease the alignment of all cavities within the filter and to increase the overall Q-factor, cavity two and four are designed as oversized $T E_{102}$ cavities. All other cavities, i.e., cavities one, three, and five, resonate at their basic $T E_{101}$ mode. The arrangement of the cavities as well as their dimensioning is shown in Fig. 1 (b). After calculating the initial dimensions, fine tuning takes place by coupling matrix extraction and tuning techniques. The Cauchy method is a common approach for the determination of the coupling matrix from simulated or measured S-parameters [11].

In the proposed design, the cross-coupling aperture described by parameter $d_{13}$ realizes the $\mathrm{TZ}$ far away from the passband while the second cross-coupling defined by parameter $d_{35}$ implements the TZ at $f_{T z, L p, 2}=-1.63$. All dimensions are shown in the caption of Fig. 1.

Three identical filters were manufactured by a high-precision CNC milling machine, the principal CAD model is shown in Fig. 1 (a). The diameter of the cutter is critical for the realization of the filter. The smallest dimension is given by the parameter $d_{13}=0.36 \mathrm{~mm}$. Therefore, a cutter of diameter $d_{c}=0.3 \mathrm{~mm}$ was chosen. Furthermore, all cavities have radii of $r=0.2 \mathrm{~mm}$ in the edges to account for the cutter radius. The filters were manufactured with the Roeders RXP400 HSC milling machine. This type of milling machine is characterized by a very high manufacturing accuracy and small tolerances. The high accuracy can mainly be achieved due to several features:

- The rotational frequency of the cutter is extremely high. E.g., according to specifications from the manufacturer, a cutter with diameter of $0.3 \mathrm{~mm}$ requires a rotational speed of 45,000 rpm.

- The milling machine contains a temperature compensation for fluctuations in the ambient temperature to increase the tool guidance in $\mathrm{x}-, \mathrm{y}-$, and $\mathrm{z}$-direction.

- Furthermore, each cutter is shrinked into the spindle which is very important to achieve a high manufacturing repeatability.

- A mechanical resonance compensation of the spindle further increases the manufacturing accuracy. If a mechanical resonance at the current rotational frequency is detected, the milling machine only works at rotational frequencies slightly below and above this resonance.

The properties of the milling process in terms of cutting speed, feed rate, and rotational speed are often defined by the manufacturer of the cutter. For the $0.3 \mathrm{~mm}$ cutter the settings as shown in Table 1 were used in dependence of the current work step.

As indicated in Fig. 1 (a), a H-plane split-block design was selected to ease the manufacturing. The whole filter structure was milled in the lower part (Fig. 1 (a)), while the upper side serves as a flat cover. Both parts are aligned with two alignment pins and are fixed with four screws. A recess close to the waveguide channel is foreseen to increase the contact pressure on the edges. Due to the good processability, brass (alloy CuZn37) was chosen as material for the manufacturing ( $\sigma_{\mathrm{CuZn} 37}=$ $15.15 \cdot 10^{6} \mathrm{~S} / \mathrm{m}$ ). Furthermore, anti-chocking flanges are provided on both sides to ease the alignment on the network analyzer (NWA). Figure 1 (d) shows a size comparison of a manufactured WR-3 filter with a 1 Euro coin (before sputtering). In 
Table 1 Basic settings of the milling machine for the manufacturing of the waveguide channel and the filter structure

\begin{tabular}{llll}
\hline & Roughing & Prefinishing & Finishing \\
\hline Cutting speed & $50 \mathrm{~m} / \mathrm{min}$ & $130 \mathrm{~m} / \mathrm{min}$ & $160 \mathrm{~m} / \mathrm{min}$ \\
Rotational speed & $45,000 \mathrm{rpm}$ & $45,000 \mathrm{rpm}$ & $45,000 \mathrm{rpm}$ \\
Depth & $30 \mu \mathrm{m}$ & $12 \mu \mathrm{m}$ & $9 \mu \mathrm{m}$ \\
Feed rate & $270 \mathrm{~mm} / \mathrm{min}$ & $203 \mathrm{~mm} / \mathrm{min}$ & $108 \mathrm{~mm} / \mathrm{min}$ \\
\hline
\end{tabular}

Fig. 2, the height profile of the cavity structure from one of the manufactured filters is shown, which was measured with a Keyence VK-X200 scanning microscope. As can be seen, each cavity has a homogeneous form along the z-axis (which corresponds to the depth of the cavities). Even the small "islands" in the center of every triplet are well manufactured and without obvious deviations. These "islands" were expected to be most problematic in the manufacturing and may lead to deviations between measurement and simulation.

\section{Measurement Results and Tolerance Analysis}

The measurement results of the three identical manufactured filters are shown in Fig. 3 in comparison with the simulation. The measurement was done by a R\&S ZVA67 network analyzer and ZC330 millimeter-wave frequency converters. The calibration was accomplished at the ports of the converters with standards short, offset short, match, and through. The reference plane therefore coincides with the flanges of the filter.

Figure 3 shows two types of simulation data. The cyan-colored curve indicates the lossless simulated filter response on which the design is based. The losses were neglected in a first step to increase the simulation speed and due to the lack of reliable data of the effective conductivity of the background material. The effective conductivity is mainly influenced by the surface resistance at the center frequency, the surface roughness, and losses which arise due to the split-block design.

As can be seen in Fig. 3, filter one is matched to $20 \mathrm{~dB}$ over the whole passband while the worst return loss value of filters two and three is around $15.5 \mathrm{~dB}$ and arises in the passband center. The insertion loss in the passband of all filters is on average $2.3 \mathrm{~dB}$, which corresponds to an unloaded Q-factor of $Q_{u} \approx 480$. The losses have three main sources:

- Finite conductivity of the base material: Due to the high frequency, the penetration depth of the electromagnetic field into the metal is very small. Assuming the proposed center frequency of $237.9 \mathrm{GHz}$ as well as the conductivity of brass, the skin depth is $\delta_{\text {brass }}=0.26 \mu \mathrm{m}$ and the surface resistance is $0.27 \Omega$.

- Surface roughness: The typical surface roughness of a milling process is in the order of $1 \mu \mathrm{m}$ [10], which exceeds the skin depth at these frequencies. The filters proposed here show a surface roughness between 1 and $1.5 \mu \mathrm{m}$. 


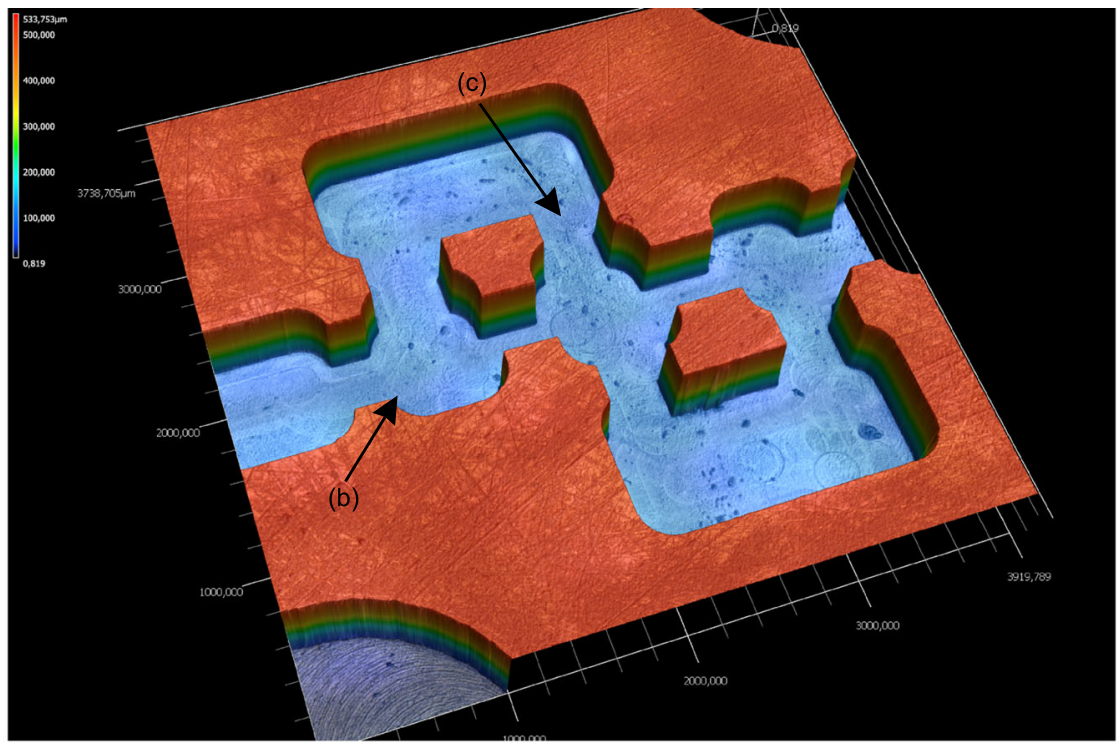

(a)

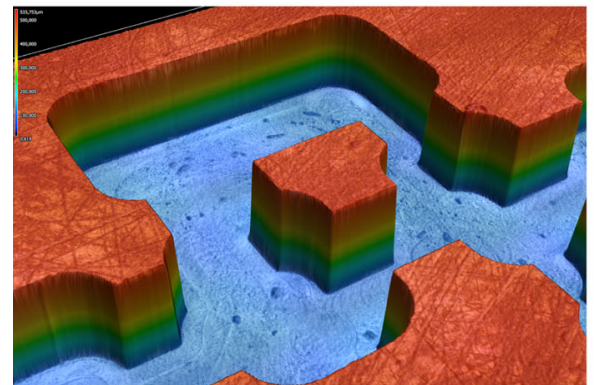

(b)

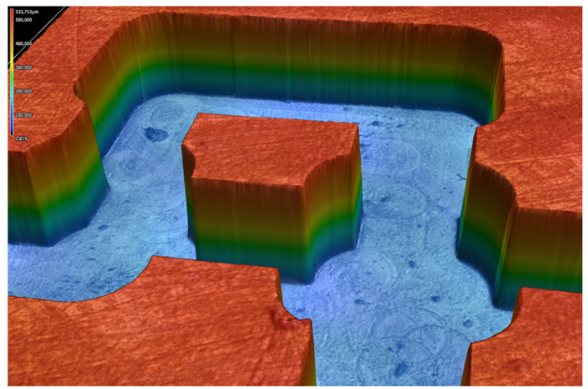

(c)

Fig. 2 (a) Complete cavity structure of one of the manufactured filters measured with a Keyence VKX200 scanning microscope (after sputtering), (b) close view of the first triplet, and (c) close view of the second triplet (compare marks in (a))

- Split-block design: The filter was designed as a H-plane cut split-block design. Surface currents are slightly disturbed as the bottom part and the cover have a finite surface roughness and are therefore not completely flat.

Figure 3 also shows the simulated S-parameters, where the conductivity was decreased until a matching with the measurement was achieved (black curve). The effective conductivity as a result of the three abovementioned effects is $\sigma_{e f f}=$ $1.8 \cdot 10^{6} \mathrm{~S} / \mathrm{m}$, which corresponds to $11.9 \%$ of the ideal DC conductivity of the used brass alloy. To classify this result, in [10] a filter at $100 \mathrm{GHz}$ in the W-band was proposed. The reduction of the conductivity from the ideal one was reported to be $18.4 \%$. 


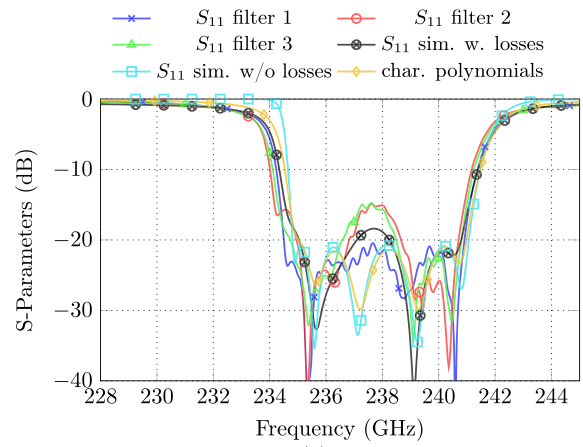

(a)

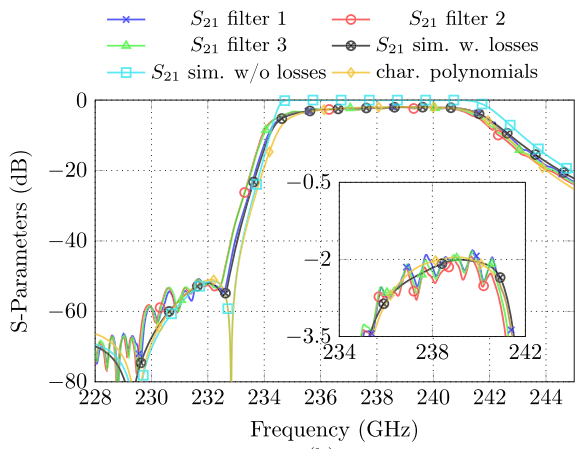

(b)

Fig. 3 Measured S-parameters of the three manufactured filters in comparison with the simulation: (a) reflection $S_{11}$ and (b) transmission $S_{21}$

As a further considerable result, the reduced conductivity leads even in the simulation to a slightly decrease from the ideal return loss in the passband at the same frequency where the measurement shows the largest deviation (Fig. 3 (a) black curve). It is further worth mentioning that the $3-\mathrm{dB}$ bandwidth is increased by roughly $1.1 \mathrm{GHz}$ which corresponds to $14 \%$ of the simulated (lossless) 3-dB bandwidth. The lower band edge is shifted by $825 \mathrm{MHz}$ whereas the upper one is only slightly shifted by $265 \mathrm{MHz}$.

In the inset of Fig. 3 (b), a slope of the insertion loss curve in dependence of the frequency can clearly be identified. This slope arises due to the finite Q-factor of the measured or simulated filters. The insertion loss is higher at lower frequencies as both TZs are placed below the passband. As a proof, a plot of the characteristic filter polynomials in which the finite Q-factor is considered is shown in Fig. 3 (b) (dark yellow curve with diamond mark). The characteristic polynomials are generated as comprehensively described in [11] and agree well with the measured data.

The measured transmission coefficient in the inset of Fig. 3 (b) shows ripples over the passband. This effect can be observed often in the literature when high-frequency components are measured, e.g., [4, 10, 14] and [15]. At these high frequencies, even small dimensional inaccuracies become visible. As the most common reason for the ripples, alignment problems between the converter and the device under test are stated in the literature. In [13], an investigation regarding connection errors at very high frequencies has taken place. Possible further reasons are tolerances in the manufacturing of the flange, especially the alignment pins. Slightly wrong positions lead to a horizontal and vertical shift of the channels between the converter and the device under test. Furthermore, at these high frequencies, a discontinuity due to manufacturing tolerances of the waveguide width $a$ and height $b$ may arise. The combination of these effects leads even in simulations to ripples in the transmission and reflection coefficient.

Furthermore, the proposed filters were manufactured by two milling machines: The first one was used to process the outer housing including the flanges whereas the waveguide channel and the cavity structure was manufactured by a much more sensitive milling machine. The filters must be re-clamped in the second milling machine, 
where the geometrical zero position must be found again. This procedure increases the risk for manufacturing tolerances at the flange.

As a conclusion of this investigation, filter one merges the design criteria in terms of return loss completely, while filters two and three show a slight degradation of the desired return loss value (15.5 $\mathrm{dB}$ at the worst position).

Based on these results, it is interesting to evaluate the proposed structure regarding manufacturing tolerances. This investigation might be useful if the milling inaccuracy in $\mathrm{x}-\mathrm{y}$-direction is known beforehand. Based on the dimensional root mean square (RMS) error, it might be possible to give a rough estimation about the lower and upper bound of the expected return loss in the passband. For this investigation, roughly 18,000 simulations were evaluated. Referring to [1], all possible dimensions of the filter from Fig. 1 (b) are varied, i.e., the diameter of each blend $d_{i j}$, the length of each cavity $l_{i}$, and their diameter $a_{i}$. The diameter of the cavities is assumed uniform in the ideal design and is therefore not highlighted in Fig. 1 (b). The feed lines are not part of this investigation. Furthermore, this study is accomplished for a lossless filter in accordance to [1].

For the simulations, a maximum allowed root mean square error (RMSE) $\mathrm{RMSE}_{\text {max }}$ is defined and for each dimension an error is randomly chosen in the range of [-RMSE $E_{\text {max }}, \mathrm{RMSE}_{\text {max }}$ ] and added to the original value. The chosen error for each parameter is the result of a normal distribution and the resulting RMSE of the filter structure is normally distributed as well. It is important to have a sufficiently large number of samples for each RMSE to conduct a comprehensive analysis. Therefore, it is desirable to distribute the 18,000 simulations equally over the preferred analysis range without taking away of the randomness which can occur during manufacturing. To create an equally distributed error in the range of RMSE $=[0,7] \mu \mathrm{m}$, the maximum allowed error RMSE $\mathrm{Rax}_{\text {max }}$ is set very low at first and is slowly increased. This is equal to the sum of shifted normal distributions which approximates an equally distributed RMSE in a certain range. As can be seen in Fig. 4, the RMSE in the 18,000 simulations is close to an equal distribution.

Subsequently, the evaluation of the return loss in the passband is a critical point to link the manufacturing inaccuracy to a degradation of the return loss level. For ideal Chebyshev or quasi-elliptical filter responses, the return loss level defines the points of worst matching in the passband. Furthermore, the band-edges of an ideal filter response are defined in terms of the return loss level. However, for de-tuned filters, these ideal conditions are not given. In practice, filters are often designed for a larger bandwidth as given by a specification to account for de-tuning effects especially at the band-edges. In order to give quantitative statements, it is assumed that the specified bandwidth $B^{\prime}$ is $80 \%$ of the original one for which the filter is designed $\left(B^{\prime} \approx 5 \mathrm{GHz}\right.$ ). The return loss can now be evaluated within the window defined by the center frequency and bandwidth, similar as proposed in [1]. As usual in practice, the worst value within this window is defined as "return loss"; therefore, the following equation holds:

$$
R L(d B)=-\left.\left[\max \left(S_{11}(d B)\right)\right]\right|_{f_{0}-B^{\prime} / 2} ^{f_{0}+B^{\prime} / 2},
$$

where $f_{0}=237.9 \mathrm{GHz}$ as defined in Section 2 . 


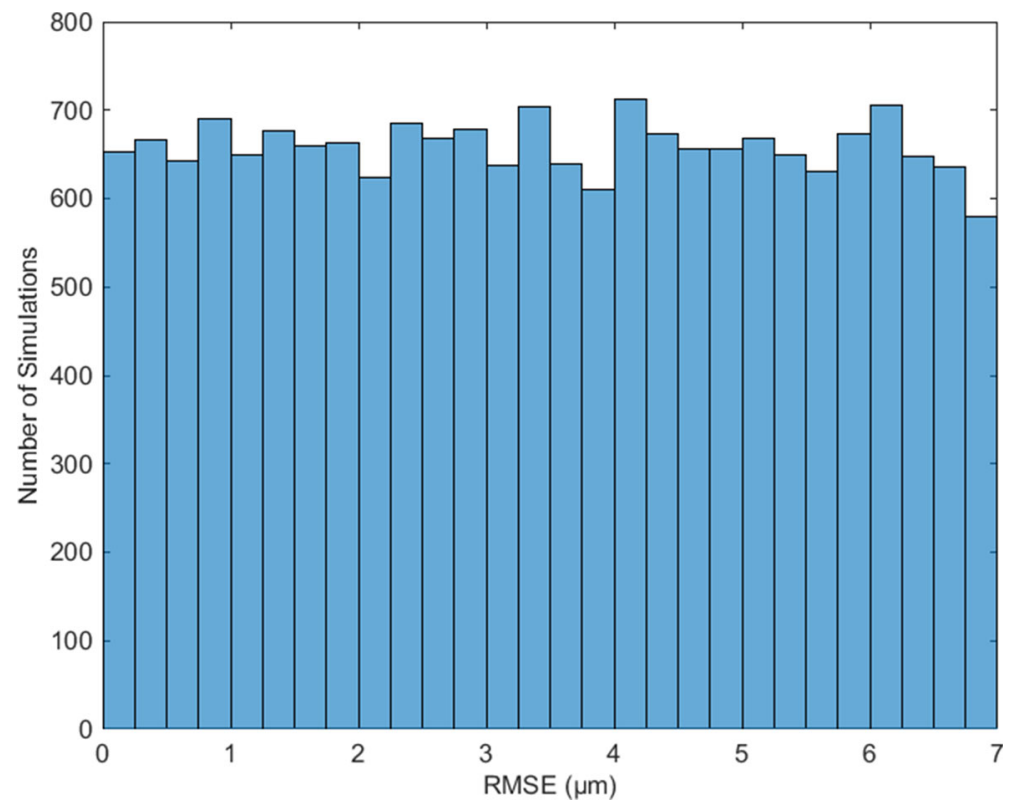

Fig. 4 Histogram showing the number of simulations in dependence of the RMSE

The result of the tolerance analysis is shown in Fig. 5. The x-axis shows the RMSE in $\mu \mathrm{m}$ while the expected return loss evaluated with Eq. 1 is plotted on the y-axis. The relative incidence normalized to the maximum value of each small column is coded by the color of the heat-map. Based on these results, it is possible to define an equation for the expected value given a RMS deviation. The corresponding curve is shown in Fig. 5 black colored. This curve is based on an exponential curve fitting as

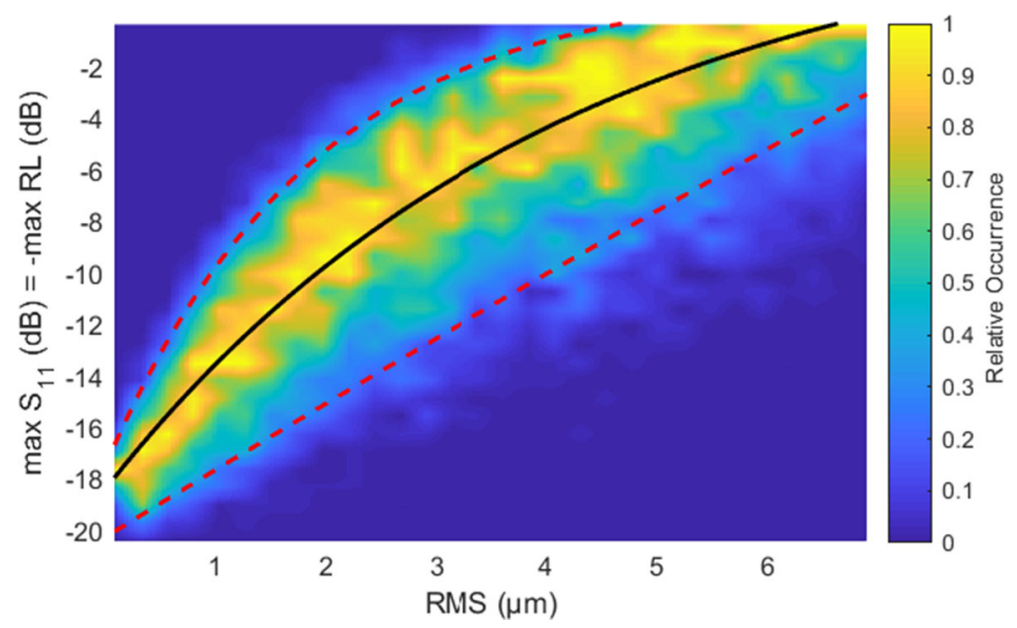

Fig. 5 Tolerance analysis in dependence of the dimensional RMSE. The return loss is evaluated with Eq. 1 
described in Eq. 2, whose coefficients are found by optimization:

$$
-R L_{f i t}(d B)=S_{11, f i t}(d B)=a \cdot e^{b \cdot \mathrm{RMS}}+c
$$

where RMS is the root mean square deviation in millimeters. The coefficients are given in Table 2. Furthermore, two additional curves are included in Fig. 5. The space between them comprises $85 \%$ of all simulated results. The upper red dashed curve defines an upper bound for the worst case, and almost $92.5 \%$ of all simulations results show a return loss level which lies below this curve. A similar statement is valid for the red dashed lower curve, and almost $92.5 \%$ of all simulations results show a return loss level which is better than defined by this curve.

As an overall result, the estimated return loss in dependency of the dimensional RMSE can be good approximated with an exponential function. If for example the root mean square deviation of a milling machine (in $\mathrm{x}$ - and y-direction) is known and given by $2.5 \mu \mathrm{m}$, the expected return loss value lies with a reliability of $85 \%$ between 5.6 and $14.7 \mathrm{~dB}$.

\section{Effect of Surface Metallization}

The aim of the following investigation is to increase the Q-factor of the measurement results in Fig. 3. The filters proposed here were sputtered with three different materials. On the one hand, the surface can be metallized with a material with a higher conductivity while on the other hand the surface roughness is reduced by the sputtering process. Both effects therefore increase the overall Q-factor. Table 3 gives an overview about which filter from the measurement in Fig. 3 is sputtered with which material and the corresponding resulting Q-factors. As sputter targets, materials with a purity of $99.95 \%$ are used. The sputter height was chosen to be maximal $0.5 \mu \mathrm{m}$, which is 3.25 times the skin depth of gold at $238 \mathrm{GHz}$. Otherwise, the layer thickness should not be chosen too high as the filter geometry and hence the electrical performance will be influenced. Please note that sputtering is an anisotropic process. Due to a partially shadowing of the cavity bottom or side walls, the sputter rate might be smaller within the filter structure compared with the flat surface of e.g. the cover.

The measurement results are shown in Fig. 6 in comparison with the S-parameters of the filters before sputtering (from Section 3). Obviously, filter one shows the smallest insertion loss after sputtering, the Q-factor increases from 480 (brass) to 660 (gold). The average insertion loss is approx. $1.6 \mathrm{~dB}$, which corresponds to an

Table 2 Coefficients for the curves in Fig. 5 and Eq. 2

\begin{tabular}{lllc}
\hline- & Coefficient & & \\
\cline { 2 - 4 } Curve & $a$ & $b$ & $c$ \\
\hline Center (black curve) & -23.35 & -203.09 & 4.804 \\
Upper (red curve) & -19.18 & -402.78 & 1.44 \\
Lower (red curve) & -169.86 & -14.56 & 149.03 \\
\hline
\end{tabular}


Table 3 Overview over the filters sputtered with different materials as well as the Q-factor before and after sputtering

\begin{tabular}{llllllll}
\hline Filter \# & $Q_{\text {brass }}$ & $I L_{\text {brass }}$ & Material & Conductivity $(\mathrm{S} / \mathrm{m})$ & $Q_{\text {sputt. }}$ & $I L_{\text {sputt. }}$ & Shift $f_{0}(\mathrm{MHz})$ \\
\hline 1 & 480 & $2.3 \mathrm{~dB}$ & Gold & $45 \cdot 10^{6}$ & 660 & $1.6 \mathrm{~dB}$ & 280 \\
2 & 480 & $2.3 \mathrm{~dB}$ & Silver & $61 \cdot 10^{6}$ & 640 & $1.65 \mathrm{~dB}$ & 490 \\
3 & 480 & $2.3 \mathrm{~dB}$ & Copper & $58 \cdot 10^{6}$ & 510 & $2.1 \mathrm{~dB}$ & 110 \\
\hline
\end{tabular}

effective conductivity of $3.2 \cdot 10^{6} \mathrm{~S} / \mathrm{m}$. The Q-factor of filter two, which is sputtered with pure silver, can be increased from 480 to 640 . As an interesting result, the third filter which is sputtered with copper shows only a very small improvement. The Qfactor increases from 480 to roughly 510 . Two reasons might be obvious: On the one hand, the surface oxidizes very fast while on the other hand an anomaly of the surface resistance of copper at high frequencies is reported in the literature $[17,18]$. Due to the coating material and the resulting smaller cavities, the center frequency of the filters is shifted slightly to higher frequencies (compare Table 3). Obviously, filter two

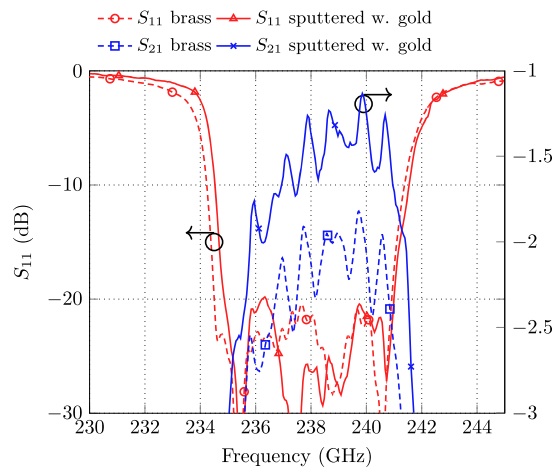

(a)

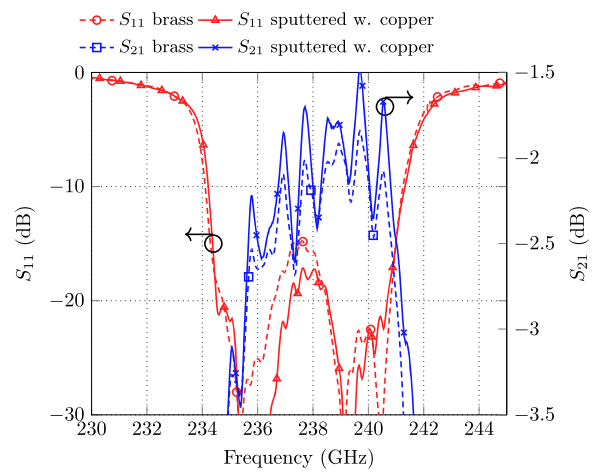

(c)

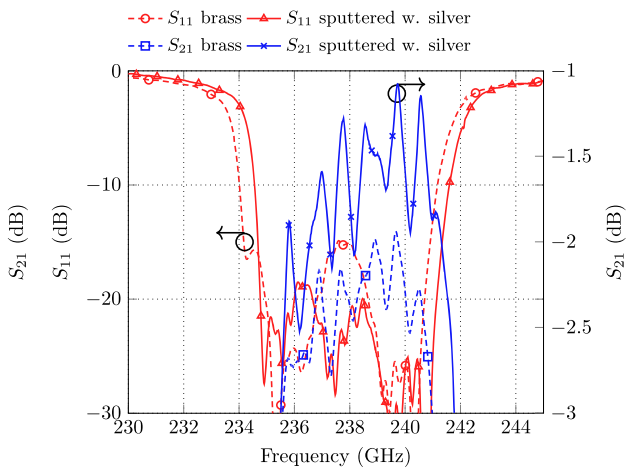

(b)

Fig. 6 Measurement results of the three filters after sputtering: (a) filter 1: gold, (b) filter 2: silver, and (c) filter 3: copper 
has a thicker sputter layer compared with filters one and three due to the increased center frequency shift.

Figure 7 shows a photograph of the sputtered filters. Usually, a very thin layer of chromium is used as adhesion agent between the basic material and the material to be sputtered. However, in the case of filter 2, the chromium layer was accidentally omitted, leading to a reduced adhesion of the silver material in the areas where a high pressure arises, i.e., at the cutting plane. The photo was taken after the first measurement has taken place. Therefore, the measurement results in Fig. 6 are not influenced by the splintered silver.

\section{Comparison}

Table 4 gives an overview over the results of this paper compared with results of related works. It is important to note that the measured insertion loss depends on the fractional bandwidth of the filter according to [12]

$$
I L(\mathrm{~dB})=4.343 \sum_{i=1}^{n} \frac{g_{i}}{F B W \cdot Q_{i}},
$$

and is therefore not a meaningful measure to compare the results. In Eq. 3, $g_{i}$ is the $i$-th component value of a related low-pass prototype, $F B W$ is the fractional bandwidth of the filter, $Q_{i}$ is the quality factor of the $i$-th resonator and $n$ is the filter order. The filters proposed here are comparably narrow-band, causing the relatively high insertion loss compared with other works. To give a meaningful comparison, estimated Q-factors are given in Table 4. These values are obtained by reconstructing the S-parameters of the referenced papers and applying the method proposed in [11].

The filters in the work proposed here show the highest Q-factor among the CNC milled filters which also have TZs at finite frequencies. The filter proposed in [19] is cut in the E-plane, which reduces the losses. However, no TZs at finite frequencies can be utilized by this filter. It is also worth mentioning that all referenced filters from

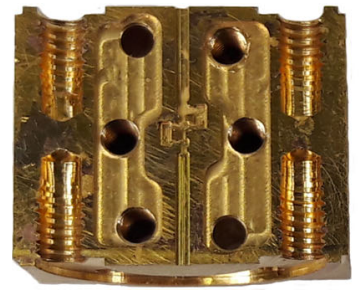

(a)

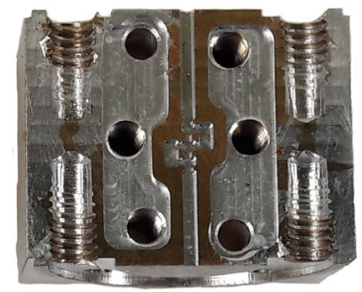

(b)

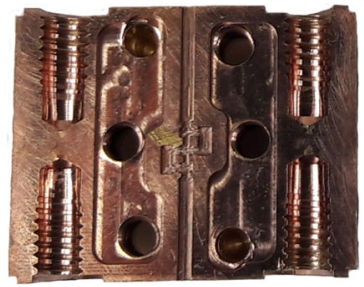

(c)

Fig. 7 Photographs of the sputtered WR-3 waveguide filters (cover removed): (a) filter 1 sputtered with gold, (b) filter 2 sputtered with silver, and (c) filter 3 sputtered with copper 
Table 4 Comparison of this work with recent related publications

\begin{tabular}{|c|c|c|c|c|c|c|c|c|}
\hline Manufact. techn. & Order & TZs & $f_{c}(\mathrm{GHz})$ & FBW & $\mathrm{IL}(\mathrm{dB})$ & $\mathrm{RL}(\mathrm{dB})$ & $Q_{u}$ & Ref \\
\hline $\mathrm{CNC} / \mathrm{H}$-cut & 4 & 2 & 257.7 & $8.77 \%(3 \mathrm{~dB})$ & $\approx 0.7$ & $>15$ & $460 *$ & {$[6]$} \\
\hline $\mathrm{CNC} / \mathrm{H}$-cut & 4 & 2 & 256.3 & $9.83 \%(3 \mathrm{~dB})$ & $\approx 0.5$ & $>15$ & $550 *$ & {$[6]$} \\
\hline CNC / E-cut & 4 & 0 & 340 & $5.29 \%$ & $\approx 0.6$ & $>20$ & $650 *$ & [19] \\
\hline CNC / H-cut & 4 & 2 & 214.6 & $9.8 \%(3 \mathrm{~dB})$ & $\approx 0.6$ & $>15$ & $450 *$ & {$[5]$} \\
\hline SU-8 & 5 & 0 & 300 & $8 \%$ & $\approx 1.6$ & $>12$ & $320 *$ & [16] \\
\hline DRIE & 4 & 2 & 270 & $1.85 \%$ & $\approx 1.5$ & $>18$ & $820 *$ & {$[8]$} \\
\hline $\mathrm{CNC} / \mathrm{H}$-cut & 5 & 2 & 237.9 & $2.64 \%$ & $\approx 1.6$ & $>20$ & 660 & Filter 1 \\
\hline $\mathrm{CNC} / \mathrm{H}$-cut & 5 & 2 & 237.9 & $2.64 \%$ & $\approx 1.65$ & $>18.5$ & 640 & Filter 2 \\
\hline
\end{tabular}

*Denotes estimated values. In case that the filter was fabricated by CNC milling, "H-" or "E- cut" denotes the manufacturing cut which is necessary for all components which are realized by abrasive manufacturing techniques

Table 4, which are manufactured by $\mathrm{CNC}$ milling, have a relatively high fractional bandwidth. The high fractional bandwidth leads to a reduced sensitivity regarding manufacturing tolerances. The filters proposed in [8] are manufactured with the deep reactive ion etching technique. Advantageously, the filter is manufactured in a chip and therefore extremely flat, avoiding losses in the feed ports. However, it is assumed that this technique has less degrees of freedom in the design process compared with the CNC milling techniques. As a result, one filter proposed here shows the highest Q-factor among the CNC milled waveguide filters, exploits two TZs positioned below the passband, and is matched to at least $20 \mathrm{~dB}$ over the whole passband. A further reduction of the losses is possible, if the feed lines are reduced in length.

\section{Conclusion}

This paper presents the design and realization of a fifth-order filter consisting of two cascaded triplets, which reveal two TZs below the passband. Three identical filters were manufactured in the WR-3 band, which enables a comparison in terms of electrical performance and geometrical manufacturing accuracy. The filters reveal similar performances and show a good agreement with the simulation. A tolerance analysis is accomplished to link a dimensional RMS error of the manufacturing technique to an expected return loss value. As all filters were manufactured in brass, they where sputtered in a second step with different materials to increase the Q-factor. An improvement of the Q-factor of $37.5 \%$ as well as $33.3 \%$ can be achieved by sputtering with gold and silver, respectively. A comparison with related works shows that the quality factor is rather good compared with related work. Furthermore, a return loss of $20 \mathrm{~dB}$ over the whole passband has been reached by one filter, which is not reported in the literature for filters which also have TZs at finite frequencies. 
Funding Information Open Access funding provided by Projekt DEAL.

Open Access This article is licensed under a Creative Commons Attribution 4.0 International License, which permits use, sharing, adaptation, distribution and reproduction in any medium or format, as long as you give appropriate credit to the original author(s) and the source, provide a link to the Creative Commons licence, and indicate if changes were made. The images or other third party material in this article are included in the article's Creative Commons licence, unless indicated otherwise in a credit line to the material. If material is not included in the article's Creative Commons licence and your intended use is not permitted by statutory regulation or exceeds the permitted use, you will need to obtain permission directly from the copyright holder. To view a copy of this licence, visit http://creativecommonshorg/licenses/by/4.0/.

\section{References}

1. Bornemann, J., Amari, S., Vahldieck, R.: A flexible S-matrix algorithm for the design of folded waveguide filters. In: European Microwave Conference, 2005. vol. 1, pp. 4-pp. IEEE (2005).

2. Cameron, R.J., Kudsia, C.M., Mansour, R.R.: Microwave Filters for Communication Systems. Wiley (2007).

3. Chen, Q., Lancaster, M., Xu, J., Tian, Y., Shang, X.: SU-8 micromachined WR-3 band waveguide bandpass filter with low insertion loss. Electronics Letters 49(7), 480-482 (Mar 2013). https://doi.org/10.1049/el.2013.0277.

4. Crowe, T.W., Foley, B., Durant, S., Hui, K., Duan, Y., Hesler, J.L.: VNA frequency extenders to 1.1 THz. In: 2011 International Conference on Infrared, Millimeter, and Terahertz Waves. IEEE (Oct 2011). https://doi.org/10.1109/irmmw-thz.2011.6105028.

5. Ding, J.Q., Shi, S.C., Zhou, K., Liu, D., Wu, W.: Analysis of 220-GHz low-loss quasi-elliptic waveguide bandpass filter. IEEE Microw. and Wireless Compon. Lett. 27(7), 648-650 (Jul 2017). https://doi.org/10.1109/1mwc.2017.2711544.

6. Ding, J.Q., Shi, S.C., Zhou, K., Zhao, Y., Liu, D., Wu, W.: WR-3 band quasi-elliptical waveguide filters using higher order mode resonance. IEEE Trans. Microw. Theory Techn. 7(3), 302-309 (May 2017).

7. Ding, J., Shi, S., Wu, W.: Cavity bandpass filters with quasi-elliptic response at $220 \mathrm{GHz}$. IEEE International Conference on Microwave and Millimeter Wave Technology (ICMMT) (Dec 2016).

8. Glubokov, O., Zhao, X., Beuerle, B., Campion, J., Shah, U., Oberhammer, J.: Micromachined multilayer bandpass filter at $270 \mathrm{GHz}$ using dual-mode circular cavities. In: 2017 IEEE MTT-S International Microwave Symposium (IMS). IEEE (Jun 2017). https://doi.org/10.1109/mwsym.2017.8058894.

9. Hong, J.S., Lancaster, M.J.: Microstrip Filters for RF/Microwave applications. John Wiley \& Sons Inc. (2001).

10. Leal-Sevillano, C.A., Montejo-Garai, J.R., Ruiz-Cruz, J.A., Rebollar, J.M.: Low-loss elliptical response filter at $100 \mathrm{GHz}$. IEEE Microw. and Wireless Compon. Lett. 22(9), 459-461 (Sep 2012). https://doi.org/10.1109/1mwc.2012.2212237.

11. Macchiarella, G.: Extraction of unloaded Q and coupling matrix from measurements on filters with large losses. IEEE Microw. and Wireless Compon. Lett. 20(6), 307-309 (Jun 2010).

12. Matthaei, G., Young, L., Jones, E.M.T.: Microwave Filters, Impedance Matching Networks and Coupling Structures. Artech House, Norwood, MA 192 (1964).

13. Oleson, C., Denning, A.: Millimeter wave vector analysis calibration and measurement problems caused by common waveguide irregularities. In: 56th ARFTG Conference Digest. IEEE (Nov 2000). https://doi.org/10.1109/arftg.2000.327428.

14. Ridler, N., Li, C.: Benchmarking electrical loss in rectangular metallic waveguide at submillimeter wavelengths. In: 2017 10th UK-Europe-China Workshop on Millimetre Waves and Terahertz Technologies (UCMMT). IEEE (Sep 2017). https://doi.org/10.1109/ucmmt.2017.8068355.

15. Shang, X., Ke, M., Wang, Y., Lancaster, M.: Micromachined WR-3 waveguide filter with embedded bends. Electronics Letters 47(9), 545 (Apr 2011). https://doi.org/10.1049/el.2011.0525.

16. Shang, X., Ke, M., Wang, Y., Lancaster, M.J.: WR-3 band waveguides and filters fabricated using SU8 photoresist micromachining technology. IEEE Trans. THz Sci. Technol. 2(6), 629-637 (Nov 2012). https://doi.org/10.1109/tthz.2012.2220136. 
17. Tischer, F.: Excess conduction losses at millimeter wavelengths. IEEE Transactions on Microwave Theory and Techniques 24(11), 853-858 (Nov 1976). https://doi.org/10.1109/tmtt.1976.1128973.

18. Tischer, F.: Experimental attenuation of rectangular waveguides at millimeter wavelengths. IEEE Transactions on Microwave Theory and Techniques 27(1), 31-37 (Jan 1979). https://doi.org/10.1109/tmtt.1979.1129554.

19. Zhang, N., Song, R., Hu, M., Shan, G., Wang, C., Yang, J.: A low-loss design of bandpass filter at the terahertz band. IEEE Microw. and Wireless Compon. Lett. 28(7), 573-575 (Jul 2018). https://doi.org/10.1109/lmwc.2018.2835650.

Publisher's Note Springer Nature remains neutral with regard to jurisdictional claims in published maps and institutional affiliations. 